\title{
IMPLICIT INTEGRATION METHOD OF SHAPE MEMORY ALLOYS CONSTITUTIVE MODEL
}

\author{
Vladimir Lj. Dunić ${ }^{1}$, Radovan B. Slavković ${ }^{2}$, Nenad M. Busarac ${ }^{1}$, Vukašin R. Slavković ${ }^{1}$ \\ and Miroslav M. Živković ${ }^{2}$ \\ ${ }^{1}$ Faculty of Engineering, University of Kragujevac \\ address \\ e-mail: \{vladimirdunic,nbusarac,vukasinsl\}@gmail.com \\ ${ }^{2}$ Faculty of Engineering, University of Kragujevac \\ address \\ e-mail: $\{$ radovan,zile\}@kg.ac.rs
}

Keywords: shape memory alloys, implicit stress integration, trial stress direction, large strain theory, governing parameter method

\begin{abstract}
Shape memory alloys (SMA) material is widely used in many applications of interest where it is necessary to recover permanent strains. There are many constitutive models which describe that phenomenon. The SMA model described by Lagoudas in [1] and [2] is modified and implemented into the PAK software package [3]. Development of this model is motivated by previous papers of Lagoudas and Auricchio groups and it considers governing parameter method applied on phenomenological behavior of SMA materials. All variables are derived to depend on effective values of stress and strain and now we have only one scalar equation to solve in the integration point. Critical thermodynamic force is also modified to match new integration method. Integration is done for the deviatoric and mean stress using trial stress direction for each time step. Difference between the return mapping algorithm and the integration in the trial deviatoric stress direction can be neglected for nearly proportional loading [2]. The second case provide possibility to solve large strain problems in the similar manner, using the same constitutive model relations. One dimensional loaded examples of super-elasticity, shape memory and thermally induced phase transformation effect proposed by Lagoudas group are verified by modified governing parameter method using the PAK software. Several large strain examples are examined under uniaxial and non-proportional loading and the results are compared with the experimental results proposed in referenced papers.
\end{abstract}




\section{INTRODUCTION}

Shape memory alloys are smart materials which have possibility to recover permanent deformations. Those deformations occur due to phase transformation between austenite and martensite. Austenite transforms to martensite by lattice shearing mechanism [4]. The phase transformation can produce two effects: superelasticity $T>A_{f}$ and the shape memory effect $T<A_{s}$, where $T$ is given temperature and $A_{s}$ and $A_{f}$ are austenit start and finish temperature. Superor pseudo- elasticity produces inelastic strains recoverable during the unloading process. The shape memory effect produces a permanent strain which can be recovered by heating [4]. The SMAs are widely used materials in the aerospace, medical and petroleum industries [5]. There are two groups of such models: micromechanics-based models and phenomenological models [1]. In the first group the information about the microstructure of the SMA are necessary for prediction, while the phenomenological models need energy potentials over the volume. In this work, we propose a model inspired by the Lagoudas theory [6]. The Lagoudas model, implemented by UMAT subroutine into Abaqus, was modified and adopted using the governing parameter method proposed by Kojic [7]. All variables are derived to depend on effective values of stress, strain and martensitic volume fraction. The critical thermodynamic force is modified to match new integration method. Integration is done for the deviatoric and mean stress using trial stress direction. Constitutive model verification is difficult due to the lack of experimental data, but some experimental results [8] are of great importance. The obtained results of numerical simulations are in close agreement with the results from literature [9] in one dimensional loaded examples of super-elasticity and shape memory. The large strain example is examined under non-proportional loading and the results are similar with the experimental results proposed in referenced papers.

\section{CONSTITUTIVE MODEL FORMULATION}

\subsection{GIBBS FREE ENERGY FUNCTION}

The constitutive model for any material can be derived from a free energy function and internal and external state variables. The phase transformation model for SMA materials formulated by Boyd and Lagoudas [10] and Lagoudas [6] is basic framework for this work. The external state variables are total stress $\sigma$ and temperature $T$. Internal state variables are the martensitic volume fraction $\xi$ and the transformation strain $\boldsymbol{e}_{t r}$. We have transformed thermodynamic potential given by Gibbs free energy function $G$ into the two different form for the forward and reverse transformation. We are starting from the basic form of Gibbs free energy given by Qidwai and Lagoudas [11]

$$
\begin{aligned}
G\left(\boldsymbol{\sigma}, T, \xi, \boldsymbol{e}_{t r}\right)= & -\frac{1}{2 \rho} \boldsymbol{\sigma}: \boldsymbol{C}^{-1}: \boldsymbol{\sigma}-\frac{1}{\rho} \boldsymbol{\sigma}:\left[\boldsymbol{\alpha}\left(T-T_{0}\right)+\boldsymbol{e}_{t r}\right]+ \\
& c\left[\left(T-T_{0}\right)-T \ln \left(\frac{T}{T_{0}}\right)\right]-s_{0} T+u_{0}+\frac{1}{\rho} f(\xi)
\end{aligned}
$$

where $T_{0}$ is a reference temperature. The function $f(\xi)$ is a transformation hardening function. The material parameters $c, u_{0}, s_{0}, \boldsymbol{C}^{-1}, \boldsymbol{\alpha}, c$ and $u_{0}$ are the effective specific heat, the effective specific internal energy, the effective specific entropy, the effective compliance tensor and the effective thermal expansion tensor, respectively. These effective material parameters can be determined using pure phases parameters and the martensitic volume fraction $\xi$ by the rule of mixture. 
Using the Legendre transformation between Gibbs free energy, the internal energy $u$ and the first and second law of thermodynamics substituted into the Clausius-Planck inequality, we obtain [6]:

$$
D_{i n t}=\boldsymbol{\sigma}: \dot{\boldsymbol{e}}_{t r}+\left(-\rho \frac{\partial G}{\partial \xi}\right) \dot{\xi} \geq 0
$$

which states that in irreversible processes the internal dissipation of energy is greater or equal zero.

\subsection{EVOLUTION EQUATIONS OF STATE VARIABLES}

The main assumption for the evolution of internal state variables is: any change in the current microstructural state of the material is strictly a result of a change in the martensitic volume fraction $[1,6,10,11]$. In that case, it is assumed that transformation strain rate is:

$$
\dot{\boldsymbol{e}_{t r}}=\boldsymbol{\Lambda}_{t r} \dot{\xi} ; \quad \boldsymbol{\Lambda}_{t r}= \begin{cases}\frac{3}{2} H \frac{\boldsymbol{S}}{\bar{S}} ; & \dot{\xi}>0 \\ H \frac{e_{t r}}{\bar{e}_{t r}} ; & \dot{\xi}<0\end{cases}
$$

where $\boldsymbol{\Lambda}_{t r}$ is the transformation tensor and $H$ is a maximum uniaxial transformation strain. The transformation tensor will be in the direction of the deviatoric stress $\boldsymbol{S}$ during the forward phase, while, during the reverse phase, it will be in the direction of transformation total strain tensor $\boldsymbol{e}_{t r}$ [6]. The deviatoric stress tensor $\boldsymbol{S}$, the effective (von Mises equivalent) stress $\bar{S}$ and the effective transformation strain $\bar{e}_{t r}$ are defined by:

$$
\boldsymbol{S}=\boldsymbol{\sigma}-\left(\sigma_{m}\right) \mathbf{1} ; \quad \bar{S}=\sqrt{\frac{3}{2} \boldsymbol{S}: \boldsymbol{S}} ; \quad \bar{e}_{t r}=\sqrt{\frac{2}{3} \boldsymbol{e}_{t r}: \boldsymbol{e}_{t r}}
$$

where $\sigma_{m}$ is mean stress and $\boldsymbol{\sigma}$ is total stress tensor.

Substituting the equation (3) into the Clausius-Planck inequality (2), we obtain:

$$
\left(\boldsymbol{\sigma}: \boldsymbol{\Lambda}-\rho \frac{\partial G}{\partial \xi}\right) \dot{\xi}=\pi \dot{\xi} \geq 0
$$

where $\pi$ is the general thermodynamic force. For the given Gibbs free energy (1), the explicit evaluation of $\pi$ is:

$$
\begin{array}{r}
\pi(\boldsymbol{\sigma}, T, \xi)=\boldsymbol{\sigma}: \boldsymbol{\Lambda}+\frac{1}{2} \boldsymbol{\sigma}: \Delta \boldsymbol{C}^{-\mathbf{1}}: \boldsymbol{\sigma}+\boldsymbol{\sigma}: \Delta \boldsymbol{\alpha}\left(T-T_{0}\right)- \\
\rho \Delta c\left[\left(T-T_{0}\right)-T \ln \left(\frac{T}{T_{0}}\right)\right]+\rho \Delta s_{0} T-\rho \Delta u_{0}-\frac{\partial f}{\partial \xi}
\end{array}
$$

The transformation function $\Phi$ is introduced by Lagoudas as [6, 11]:

$$
\Phi= \begin{cases}\pi-Y ; & \dot{\xi}>0 \\ -\pi-Y & \dot{\xi}<0\end{cases}
$$

where $Y$ is threshold value. The transformation function $\Phi$ should satisfy the condition $\Phi=0$ during phase transformation. It represents transformation surfaces for $0<\xi<1$ and the two boundary surfaces for $\xi=0$ and $\xi=1$ of each transformation surface family. 
Different hardening functions $f(\xi)$ can be used to describe interaction between the austenit and the martensit. Cosine model proposed by Liang and Rogers is given as[6, 12]:

$$
f(\xi)= \begin{cases}\int_{0}^{\xi}-\frac{\rho \Delta s_{0}}{a_{c}^{M}}[\pi-\arccos (2 \xi-1)] d \xi+\left(\mu_{1}^{c}+\mu_{2}^{c}\right) \xi ; & \dot{\xi}>0 \\ \int_{0}^{\xi}-\frac{\rho \Delta s_{0}}{a_{c}^{A}}[\pi-\arccos (2 \xi-1)] d \xi+\left(\mu_{1}^{c}+\mu_{2}^{c}\right) \xi ; & \dot{\xi}<0\end{cases}
$$

In above equations $a_{c}^{M}, a_{c}^{A}, \mu_{1}^{c}, \mu_{2}^{c}$ are transformation strain hardening parameters. Introducing equation (8) into the (6) and using the parameters for the cosine model given by Liang and Rogers [12] using the previous equations in (7), the final form of the transformation function is obtained as:

$$
\Phi=\left\{\begin{array}{c}
H \bar{S}+\frac{1}{2}\left(\frac{1}{3} \bar{S}^{2}\left(\frac{1}{G^{M}}-\frac{1}{G^{A}}\right)+3 \sigma_{m}^{2}\left(\frac{1}{c_{m}^{M}}-\frac{1}{c_{m}^{A}}\right)\right)+3 \sigma_{m}\left(\alpha^{M}-\alpha^{A}\right) \Delta T+ \\
\rho \Delta s_{0}\left(T-M_{s}\right)-\rho \Delta s_{0} \frac{M_{s}-M_{f}}{\pi}[\arccos (2 \xi-1)-\pi] ; \quad \dot{\xi}>0 \\
-\boldsymbol{S}^{*}:^{t} \boldsymbol{e}_{t r} \frac{H}{\bar{t}_{\bar{e}} t r}+3 G \Delta \xi H^{2}-\frac{1}{2}\left(\frac{1}{3} \bar{S}^{2}\left(\frac{1}{G^{M}}-\frac{1}{G^{A}}\right)+3 \sigma_{m}^{2}\left(\frac{1}{c_{m}^{M}}-\frac{1}{c_{m}^{A}}\right)\right)- \\
3 \sigma_{m}\left(\alpha^{M}-\alpha^{A}\right) \Delta T-\rho \Delta s_{0}\left(T-A_{f}\right)+ \\
\rho \Delta s_{0} \frac{A_{f}-A_{s}}{\pi}[\arccos (2 \xi-1)-\pi] ; \quad \dot{\xi}<0
\end{array}\right.
$$

\section{NUMERICAL IMPLEMENTATION OF STRESS INTEGRATION PROCEDURE}

\subsection{STRESS INTEGRATION}

The most common strategy for implicit stress integration procedure for the rate independent models is the return mapping [7]. It consists of two steps: (a) prediction of the trial elastic state (elastic predictor), and (b) stress correction due to the phase transformation (inelastic corrector). The trial elastic deviatoric stress ${ }^{t+\Delta t} \boldsymbol{S}_{*}^{E}$ and the mean stress ${ }^{t+\Delta t} \sigma_{m}^{E}$ are calculated with the assumption that there is no phase transformation in the time step:

$$
{ }^{t+\Delta t} \boldsymbol{S}_{*}^{E}=2^{t+\Delta t} G^{t+\Delta t} \boldsymbol{e}_{*}^{E^{\prime}} ; \quad{ }^{t+\Delta t} \sigma_{m}^{E}={ }^{t+\Delta t} c_{m}\left({ }^{t+\Delta t} e_{* m}^{E}-{ }^{t+\Delta t} e^{t h}\right)
$$

In the case of the small strains, the trial elastic deviatoric strain ${ }^{t+\Delta t} \boldsymbol{e}_{*}^{E^{\prime}}$, the trial elastic mean strain ${ }^{t+\Delta t} e_{* m}^{E}$ and thermal strain ${ }^{t+\Delta t} e^{t h}$ are:

$$
{ }^{t+\Delta t} \boldsymbol{e}_{*}^{E^{\prime}}={ }^{t+\Delta t} \boldsymbol{e}-e_{m} \boldsymbol{I}-{ }^{t} \boldsymbol{e}^{t r} ; \quad{ }^{t+\Delta t} e_{* m}^{E}=\frac{1}{3} \sum_{k=1}^{3}{ }^{t+\Delta t} e_{k k} ; \quad{ }^{t+\Delta t} e^{t h}={ }^{t+\Delta t} \alpha \Delta T
$$

where ${ }^{t+\Delta t} G$ is shear modulus, ${ }^{t+\Delta t} c_{m}$ is $1 / 3$ of bulk modulus $K$ and ${ }^{t+\Delta t} \alpha$ is coefficient of thermal expansion, which depend on $\xi$. The total strain at the end of time step and the transformation at the start of the time step are ${ }^{t+\Delta t} \boldsymbol{e}$ and ${ }^{t} \boldsymbol{e}^{t r}$, respectively.

\subsection{ITERATIVE SOLUTION OF TRANSFORMATION FUNCTION EQUATION}

This work is based on developments from the literature and it is inspired by Kojic and Bathe [7], Simo and Hughes [13] and Qidwai and Lagoudas [1, 11]. The total strain $e$ and temperature $T$ are given variables and the SMA constitutive model outputs total stress $\boldsymbol{\sigma}$ and updates internal variables. The problem is defined by total strain relation given by Lagoudas in [6], the flow rule (3) and the transformation function (9). First, a thermoelastic prediction assumes that the transformation strain increment $\Delta^{t+\Delta t} \boldsymbol{e}_{t r}$ is zero (11). The governing parameter method has 
been used instead the convex cutting plane algorithm. The difference between them is in the direction of the transformation correction. In the convex cutting plane algorithm, direction of the transformation correction is changed during the iteration, while, the governing parameter method assumes trial deviatoric stress direction (10) from the beginning of the time step.

Increment of transformation strain in time step is:

$$
\Delta^{t+\Delta t} \boldsymbol{e}_{t r}=\Delta^{t+\Delta t} \xi^{(k)} \Lambda\left({ }^{t+\Delta t} \boldsymbol{S}_{*}^{E}\right)
$$

The algorithm begins at iteration $k=0$ with the thermoelastic prediction (10) with assumption that there is no evolution in the internal variables ${ }^{t+\Delta t} \boldsymbol{e}_{t r}^{(0)}={ }^{t} \boldsymbol{e}_{t r}$ and ${ }^{t+\Delta t} \xi^{(0)}={ }^{t} \xi$ [6].

The transformation function is calculated with trial stress and the condition ${ }^{t+\Delta t} \Phi^{(0)} \leq 0$ is checked for violation [1]. If there is no violation, the elastic solution is accepted as final. If ${ }^{t+\Delta t} \Phi^{(0)} \leq 0$ is violated, correction of the solution should satisfy $\Phi=0$. For that purpose it is possible to find solution of the non-linear equation (9) by various methods like Newton-Raphson method or bisections.

By application of chain rule to the transformation function (9) for the next iteration is:

$$
{ }^{t+\Delta t} \Phi^{(k)}+d^{t+\Delta t} \Phi^{(k)}={ }^{t+\Delta t} \Phi^{(k+1)}={ }^{t+\Delta t} \Phi^{(k)}+\frac{\partial^{t+\Delta t} \Phi^{(k)}}{\partial \xi} d^{t+\Delta t} \xi^{(k)} \simeq 0
$$

Since, the transformation function does not depend on change of stress direction, using the Newton-Raphson iterative scheme we can calculate increment of martensitic volume fraction $\xi$ as:

$$
d^{t+\Delta t} \xi^{(k)}=-\frac{{ }^{t+\Delta t} \Phi^{(k)}}{\frac{\partial^{t+\Delta t} \Phi^{(k)}}{\partial \xi}}
$$

During the iteration process, the step increment of martensitic volume fraction and the martensitic volume fraction are updated as:

$$
\Delta^{t+\Delta t} \xi^{(k+1)}=\Delta^{t+\Delta t} \xi^{(k)}+d^{t+\Delta t} \xi^{(k)} ; \quad{ }^{t+\Delta t} \xi={ }^{t} \xi+\Delta^{t+\Delta t} \xi^{(k+1)}
$$

The iterative scheme continues until ${ }^{t+\Delta t} \Phi^{(k+1)}$ is smaller than some tolerance or ${ }^{t+\Delta t} \xi^{(0)}$ reaches a boundary values of 0 or 1 . Finally, with known value of martensitic volume fraction ${ }^{t+\Delta t} \xi$, we calculate deviatoric stress at the end of time step as:

$$
{ }^{t+\Delta t} \boldsymbol{S}^{E}=\left\{\begin{array}{c}
{ }^{t+\Delta t} \bar{S}^{E}{ }^{t+\Delta t}{ }^{t+\Delta t} \boldsymbol{S}_{*}^{E} ; \quad \dot{\xi}>0 \\
{ }^{t+\Delta t} \boldsymbol{S}_{*}^{E}-2^{t+\Delta t} G H \Delta^{t+\Delta t} \xi^{t}{ }^{t}{ }^{t} \bar{e}_{\bar{e}_{t r}} ; \quad \dot{\xi}<0
\end{array}\right.
$$

where effective Von Mises deviatoric stress is calculated as:

$$
{ }^{t+\Delta t} \bar{S}=\left\{\begin{array}{cc}
{ }^{t+\Delta t} \bar{S}_{*}^{E}-3^{t+\Delta t} G H \Delta^{t+\Delta t} \xi ; \quad \dot{\xi}>0 \\
\sqrt{{ }^{t+\Delta t} \bar{S}_{*}^{E}-6^{t+\Delta t} G H \Delta^{t+\Delta t} \xi \frac{{ }^{t+\Delta t} \boldsymbol{S}_{*}{ }^{t} \boldsymbol{e}_{t r}}{{ }^{t} \bar{e}_{t r}}+9^{t+\Delta t} G H \Delta^{t+\Delta t} \xi^{t} \bar{e}_{t r}} ; \quad \dot{\xi}<0
\end{array}\right.
$$

The total stress and the transformation strain (using the equation (12)), at the end of time step now can be calculated as:

$$
{ }^{t+\Delta t} \boldsymbol{\sigma}={ }^{t+\Delta t} \boldsymbol{S}+{ }^{t+\Delta t} \sigma_{m} \boldsymbol{I} ; \quad{ }^{t+\Delta t} \boldsymbol{e}_{t r}={ }^{t} \boldsymbol{e}_{t r}+\left\{\begin{array}{cc}
\frac{3 H \Delta^{t+\Delta t} \xi^{(k+1)}}{{ }^{t+\Delta t} \bar{S}^{t+\Delta t} \boldsymbol{S} ;} \quad \dot{\xi}>0 \\
\frac{H \Delta^{t+\Delta t} \xi^{(k+1)}}{{ }^{t} \bar{e}_{t r}} \boldsymbol{e}_{t r} ; \quad \dot{\xi}<0
\end{array}\right.
$$




\subsection{APPLICATION TO LARGE STRAIN THEORY}

For the large strain theory, we are using basic assumption of multiplicative decomposition of total deformation gradient on elastic and inelastic part ${ }_{0}^{t} \boldsymbol{F}={ }_{0}^{t} \boldsymbol{F}^{E t}{ }_{0}^{t} \boldsymbol{F}^{t r}$, where ${ }_{0}^{t} \boldsymbol{F}^{t r}$ corresponding to deformation from reference to local stress free configuration and ${ }_{0}^{t} \boldsymbol{F}^{E}$ from local stress free to current configuration.

The trial elastic left Cauchy-Green deformation tensor ${ }^{t+\Delta t} \boldsymbol{B}_{*}^{E}$ can be calculated using saved data for ${ }^{t} \boldsymbol{B}^{E}$ or ${ }^{t} \boldsymbol{C}_{t r}^{-1}$ from previous time step as [14]:

$$
{ }^{t+\Delta t} \boldsymbol{B}_{*}^{E}={ }^{t+\Delta t}{ }_{t} \overline{\boldsymbol{F}}^{t} \boldsymbol{B}^{E^{t+\Delta t}}{ }_{t} \overline{\boldsymbol{F}}^{T} \quad \text { or } \quad{ }^{t+\Delta t} \boldsymbol{B}_{*}^{E}={ }_{0}^{t+\Delta t} \overline{\boldsymbol{F}}^{t} \boldsymbol{C}_{t r}^{-1}{ }_{0}^{t+\Delta t} \overline{\boldsymbol{F}}^{T}
$$

where the left Cauchy-Green elastic strain tensor is ${ }^{t} \boldsymbol{B}^{E}={ }_{0}^{t} \boldsymbol{F}^{E t}{ }_{0} \boldsymbol{F}^{E^{T}}$ and the right CauchyGreen inelastic strain tensor is ${ }^{t} \boldsymbol{C}^{t r}={ }_{0}^{t} \boldsymbol{F}^{t r}{ }_{0}^{t} \boldsymbol{F}^{t r}$. The modified relative and total deformation gradient are ${ }_{t}^{t+\Delta t} \overline{\boldsymbol{F}}$ and ${ }_{0}^{t+\Delta t} \overline{\boldsymbol{F}}[7,13]$.

In the case of logarithmic strains, the trial elastic deviatoric strain ${ }^{t+\Delta t} \boldsymbol{e}_{*}^{E^{\prime}}$ is:

$$
{ }^{t+\Delta t} \boldsymbol{e}_{*}^{E^{\prime}}=\sum_{k=1}^{3} \ln \left({ }^{t+\Delta t} \lambda_{* k}^{\prime E}\right){ }^{t+\Delta t} \boldsymbol{p}_{k}^{E t+\Delta t} \boldsymbol{p}_{k}^{E}
$$

where ${ }_{0}^{t+\Delta t} \lambda_{* k}^{E^{\prime}}$ are the trial principal stretches and ${ }^{t+\Delta t} \boldsymbol{p}_{k}^{E}$ are the corresponding principal directions of the tensor ${ }^{t+\Delta t} \boldsymbol{B}_{*}^{E}$. In the case of Green-Lagrange strain tensor, the trial elastic deviatoric strain ${ }^{t+\Delta t} \boldsymbol{e}_{*}^{E^{\prime}}$ is:

$$
{ }^{t+\Delta t} \boldsymbol{e}_{*}^{E^{\prime}}=\frac{1}{2}\left({ }^{t+\Delta t} \boldsymbol{B}_{*}^{E}-\boldsymbol{I}\right)
$$

The trial elastic mean strain ${ }^{t+\Delta t} e_{* m}^{E}$ is:

$$
{ }^{t+\Delta t} e_{* m}^{E}=\frac{1}{3} \ln \left[\operatorname{det}\left({ }_{0}^{t+\Delta t} \boldsymbol{F}\right)\right]
$$

For known trial elastic state, the same integration procedure described for small strain case is applied for large strains.

Update of the left Cauchy-Green tensor ${ }^{t+\Delta t} \boldsymbol{B}^{E}$ for the logarithmic strains is done as [7, 14]

$$
{ }^{t+\Delta t} \boldsymbol{B}^{E}=\sum_{3}^{k=1} \exp \left(2^{t+\Delta t} e_{k}^{{ }^{E}}\right){ }^{t+\Delta t} \boldsymbol{p}_{k}^{E t+\Delta t} \boldsymbol{p}_{k}^{E}
$$

where ${ }^{t+\Delta t} e_{k}^{\prime E}$ are elastic deviatoric strains in the principal directions calculated as:

$$
{ }^{t+\Delta t} e_{k}^{\prime E}=\frac{{ }^{t+\Delta t} S_{k}}{2^{t+\Delta t} G}
$$

Here, ${ }^{t+\Delta t} S_{k}$ are deviatoric stresses components in the principal directions ${ }^{t+\Delta t} \boldsymbol{p}_{k}^{E}$ [7, 14]. For the case of Green-Lagrange strain tensor, the left Cauchy-Green tensor ${ }^{t+\Delta t} \boldsymbol{B}^{E}$ is:

$$
{ }^{t+\Delta t} \boldsymbol{B}^{E}=2\left({ }^{t+\Delta t} \boldsymbol{e}^{E}+\boldsymbol{I}\right)
$$


where ${ }^{t+\Delta t} \boldsymbol{e}^{E}$ total elastic strain tensor is:

$$
{ }^{t+\Delta t} \boldsymbol{e}^{E}=\frac{{ }^{t+\Delta t} \boldsymbol{S}}{2^{t+\Delta t} G}+e_{m} \boldsymbol{I}
$$

The transformation strain is calculated using the right Cauchy-Green transformation strain tensor:

$$
{ }^{t+\Delta t} \boldsymbol{C}_{t r}^{-1}={ }_{0}^{t+\Delta t} \overline{\boldsymbol{F}}^{-1} t+\Delta t \boldsymbol{B}^{E}{ }_{0}^{t+\Delta t} \overline{\boldsymbol{F}}^{-T}
$$

The transformation strain ${ }^{t+\Delta t} \boldsymbol{e}_{t r}$, in logarithmic strain case and for the Green-Lagrange strain tensor, at the end of time step is:

$$
{ }^{t+\Delta t} \boldsymbol{e}_{t r}=\sum_{k=1}^{3} \ln \left({ }^{t+\Delta t} \lambda_{l}^{t r}\right){ }^{t+\Delta t} \boldsymbol{q}_{l}^{t r t+\Delta t} \boldsymbol{q}_{l}^{t r} ; \quad{ }^{t+\Delta t} \boldsymbol{e}_{t r}=\frac{1}{2}\left({ }^{t+\Delta t} \boldsymbol{C}_{t r}-\boldsymbol{I}\right)
$$

where ${ }^{t+\Delta t} \lambda_{l}^{t r}$ are the principal stretches and ${ }^{t+\Delta t} \boldsymbol{q}_{l}^{t r}$ are the principal directions of the tensor ${ }^{t+\Delta t} \boldsymbol{C}_{t r}$.

\section{NUMERICAL EXAMPLES}

Comparison with the numerical and experimental examples from literature is used to demonstrate accuracy of proposed model. Two uniaxial and one multiaxial non-proportional loading example are presented in this work. Material parameters are adopted according to data given in literature $[1,9]$. The material parameters required for calculation are the Youngs moduli for austenite and martensite $E_{A}$ and $E_{M}$, thermal expansion coefficients $\alpha_{A}$ and $\alpha_{M}$, martensite start and finish and austenite start and finish temperatures at zero stress $M_{0}^{s}, M_{0}^{f}, A_{0}^{s}$ and $A_{0}^{f}$, maximum transformation strain $\mathrm{H}$ and austenite and martensite stress influence coefficients $\rho \Delta s_{A}$ and $\rho \Delta s_{M}$.

\subsection{Uniaxial tests}

Two uniaxial tests are done at different temperatures to show pseudo-elasticity effect and shape memory effect. Results are compared with the literature [1,9] and the good agreement was obtained. Both tests were done using the same model of 3D unit cube made of "NiTinol" alloy. The boundary and loading conditions and the material parameters are taken according to literature [9]. The model consists of two equal finite elements. The material parameters from [9], used in those examples, are given in Table 1.

Table 1: Material parameters of Nickel-Titanium alloys used in uniaxial examples [9]

\begin{tabular}{cccc}
\hline \hline$E_{A}$ & $E_{M}$ & $\alpha_{A}$ & $\alpha_{M}$ \\
$70.000 \mathrm{MPa}$ & $30.000 \mathrm{MPa}$ & $22.0 \cdot 10^{-6} K^{-1}$ & $22.0 \cdot 10^{-6} K^{-1}$ \\
$M_{0 s}$ & $M_{0 f}$ & $A_{0 s}$ & $A_{0 f}$ \\
$291 K$ & $271 K$ & $295 K$ & $315 K$ \\
$H$ & $\nu$ & $\rho \Delta s_{A}$ & $\rho \Delta s_{M}$ \\
0.05 & 0.33 & $-0.35 \mathrm{MPa} K^{-1}$ & $-0.35 \mathrm{MPa} K^{-1}$ \\
\hline
\end{tabular}




\subsubsection{Pseudo-elasticity example}

The first example describes the superelastic effect of the SMAs. The material is on constant temperature $325 \mathrm{~K}$, which is higher than the austenitic finish temperature $A_{0 f}=315 \mathrm{~K}$. The cube is loaded in uniaxial tension stress $\sigma=600 \mathrm{MPa}$ in 100 steps until full martensitic phase is achieved. The material is then unloaded in 100 equal steps to stress free configuration. As it can be seen in Figure 1, all transformation strains were fully recovered.

\subsubsection{Shape memory effect example}

The second example demonstrates the shape memory effect. A temperature was $295 K$ which is between the austenitic $A_{0 s}$ and martensitic $M_{0 s}$ start temperature. The uniaxial tension $\sigma=$ $600 \mathrm{MPa}$ was applied in 100 steps until the full transformation strain is achieved and than the cube was relaxed in another 100 steps to zero stress. After the relaxation, there were residual strains and the material was in the martensitic phase. Than, the cube was heated above the austentic finish temperature to $350 K$ in 100 steps and the transformation strains are completely recovered as it is given in Figure 2.

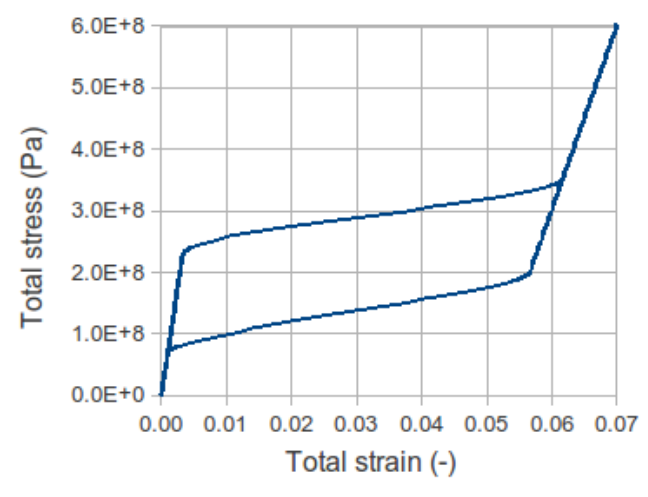

Figure 1: Stress-strain diagram for super-elasticity example

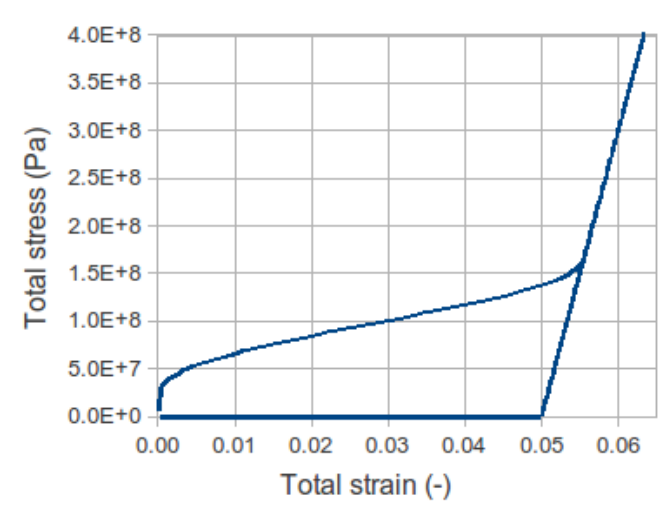

Figure 2: Stress-strain diagram for shape memory effect example

\subsection{Large strain non-proportional example}

This examples describes the model prediction for non-proportional loading with superelasticity effect. The test temperature is set to $308 \mathrm{~K}$ which is $25 \mathrm{~K}$ more than austenitic finish temperature $A_{0 s}=283 \mathrm{~K}$, so superelastic behaviour is expected. This example is experimentally examined and the obtained data are available in literature $[1,2]$. Tension-torsion experiment of thin wall specimen [8] made of CuAlZnMn alloy is used for this purpose. Axial stress of $240 \mathrm{MPa}$ and then the shear stress of $200 \mathrm{MPa}$ were applied combined at the same side of cube, while the opposite side is fully constrained. After that, first tension was unloaded and than shear strain were recovered. Material parameters that were used for this simulation are used from the literature [1]. 
Table 2: Material parameters of CuAlZnMn [8] alloy used in non-proportional example [1]

\begin{tabular}{cccc}
\hline \hline$E_{A}$ & $E_{M}$ & $\alpha_{A}$ & $\alpha_{M}$ \\
$30.000 \mathrm{MPa}$ & $10.000 \mathrm{MPa}$ & $10.0 \cdot 10^{-6} K^{-1}$ & $10.0 \cdot 10^{-6} K^{-1}$ \\
$M_{0 s}$ & $M_{0 f}$ & $A_{0 s}$ & $A_{0 f}$ \\
$278 K$ & $183 K$ & $188 K$ & $283 K$ \\
$H$ & $\nu$ & $\rho \Delta s_{A}$ & $\rho \Delta s_{M}$ \\
0.035 & 0.30 & $-0.28 \mathrm{MPa} K^{-1}$ & $-0.28 \mathrm{MPa} K^{-1}$ \\
\hline
\end{tabular}

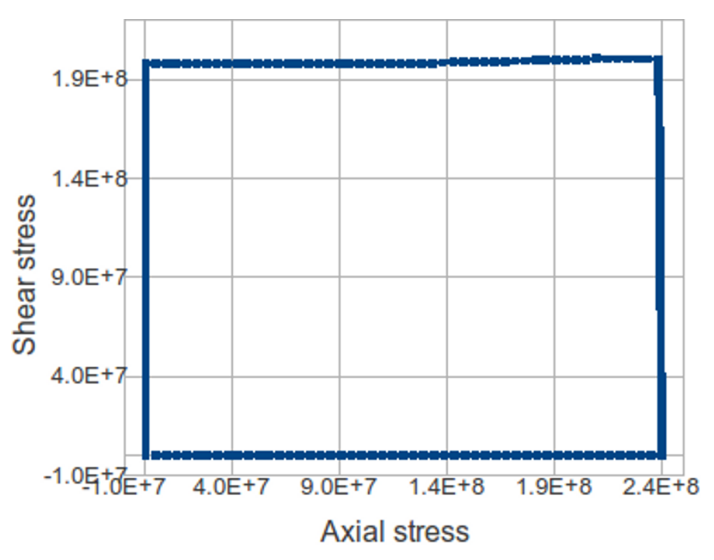

Figure 3: Axial-shear stress diagram for non proportional example

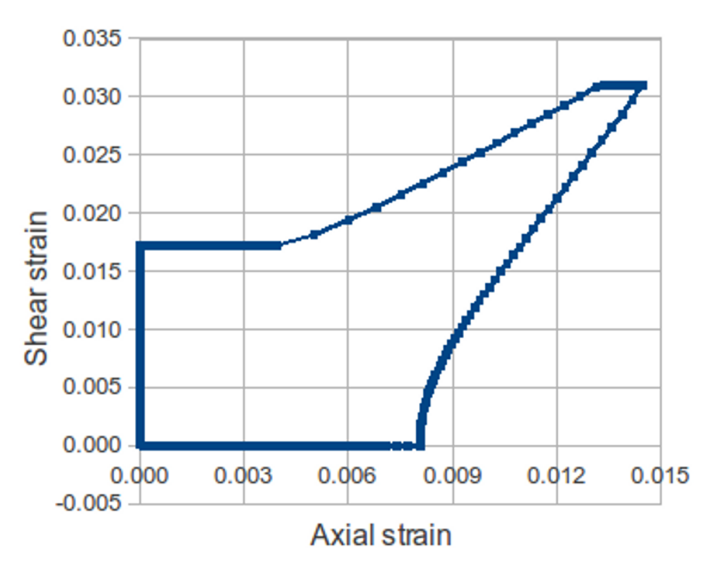

Figure 4: Axial-shear strain diagram for non proportional example

Multiaxial tension-torsion non-proportional loading path is compared with the experimental results [8] and numerical results from the literature [1, 2, 4].

\section{CONCLUSIONS}

The theory presented by Lagoudas has been modified. The total stress is decomposed on deviatoric and mean part and the stress integration is done in direction of the trial deviatoric stress. The solutions are similar to one obtained by return mapping algorithm. Instead of solving transformation function with several dependent variables, we have to solve only one scalar non-linear equation by Newton-Raphson method or using bisections. In the non-proportional example results are qualitatively similar to experimental results obtained by Šittner [8]. That change gave possibility to extend small strain theory and solve examples with large strains. Introducing multiplicative decomposition of deformation gradient and Green-Lagrange or logarithmic strain tensor, the same solution procedure can be used to find solution.

\section{REFERENCES}

[1] D.Lagoudas, D.Hartl, Y.Chemisky, L.Machado, P.Popov, Constitutive model for the numerical analysis of phase transformation in polycrystalline shape memory alloys, International Journal of Plasticity, 32-33, 155-183, 2012.

[2] J.Arghavani, F.Auricchio, R.Naghdabadi, A.Reali, S.Sohrabpour, A 3-D phenomenological constitutive model for shape memory alloys under multiaxial loadings, International Journal of Plasticity, 26, 7, 976-991, 2010. 
[3] M.Kojić, R.Slavković, M.Živković, N.Grujović, PAK-S Program for FE Structural Analysis, User Manual, Faculty of Mechanical Engineering, University of Kragujevac, Kragujevac, 2013.

[4] M.Panico, L.C.Brinson, A three-dimensional phenomenological model for martensite reorientation in shape memory alloys, Journal of the Mechanics and Physics of Solids, 55, 11, 2491-2511, 2007.

[5] D.J.Hartl, G.Chatzigeorgiou, D.C.Lagoudas, Three-dimensional modeling and numerical analysis of rate-dependent irrecoverable deformation in shape memory alloys, International Journal of Plasticity, 26, 10, 1485-1507, 2010.

[6] D.C. Lagoudas, Shape Memory Alloys: Modeling and Engineering Applications, Springer, 2010.

[7] M.Kojić, K.J.Bathe, Inelastic Analysis of Solids and Structures, 1st edition. Computational Fluid and Solid Mechanics, Springer-Verlag Berlin and Heidelberg GmbH KG, Berlin, 2005.

[8] P.Šittner, Y.Hara, M.Tokuda, Experimental study on the thermoelastic martensitic transformation in shape memory alloy polycrystal induced by combined external forces, Metallurgical and Materials Transactions A, 26, 10, 1073-5623, 1995.

[9] D.Lagoudas, Z.Bo, M.Qidwai, P.Entchev, SMA UM: User Material Subroutine for Thermomechanical Constitutive Model of Shape Memory Alloys, Texas A\&M University, College Station, TX, March 2003.

[10] J.G. Boyd, D.C. Lagoudas, A thermodynamical constitutive model for shape memory materials. Part I. The monolithic shape memory alloy, International Journal of Plasticity, 12 , 6, 805-842, 1996.

[11] M.A.Qidwai, D.C.Lagoudas, Numerical implementation of a shape memory alloy thermomechanical constitutive model using return mapping algorithms, International Journal for Numerical Methods in Engineering, 47, 6, 1097-0207, 2000.

[12] D.Lagoudas, Z.Bo, M.Qidwai, A unified thermodynamic constitutive model for SMA and finite element analysis of active metal matrix composites, Mechanics of Composite Materials and Structures, 3,2 1075-9417, 1996.

[13] J.C.Simo, T.J.R.Hughes, Computational Inelasticity, Interdisciplinary applied mathematics: Mechanics and materials, Springer, 1998.

[14] M.Kojić, I.Vlastelica, M.Živković, Implicit stress integration procedure for small and large strains of the Gurson material model, International Journal for Numerical Methods in Engineering, 53, 12, 1097-0207, 2002. 